\title{
FURTHER RESULTS ON VARIANCES OF LOCAL STEREOLOGICAL ESTIMATORS
}

\author{
ZBYNĚK PAWLAS ${ }^{1}$ AND EVA B. VEDEL JENSEN ${ }^{2}$ \\ ${ }^{1}$ Department of Probability and Mathematical Statistics, Faculty of Mathematics and Physics, Charles \\ University, Sokolovská 83, 18675 Prague, Czech Republic, ${ }^{2}$ Department of Mathematical Sciences, University \\ of Aarhus, Ny Munkegade, DK-8000 Aarhus C, Denmark \\ e-mail: pawlas@karlin.mff.cuni.cz,eva@imf.au.dk \\ (Accepted October 9, 2006)
}

\begin{abstract}
In the present paper the statistical properties of local stereological estimators of particle volume are studied. It is shown that the variance of the estimators can be decomposed into the variance due to the local stereological estimation procedure and the variance due to the variability in the particle population. It turns out that these two variance components can be estimated separately, from sectional data. We present further results on the variances that can be used to determine the variance by numerical integration for particular choices of particle shapes.
\end{abstract}

Keywords: local stereology, marked point process, model-based setting, star-shaped set, variance.

\section{INTRODUCTION}

One of the important unsolved problems in stereology concerns the stereological estimation of particle size distributions without specific assumptions about particle shape. It has been known for some time how to estimate stereologically the mean particle volume for particles of varying shape, $c f$. Jensen (1998). The resulting distribution of estimated particle volumes has been used as an estimate of the distribution of the true particle volumes. It is clearly important to be able to judge when such a procedure is justified.

The particular case of estimating the volumeweighted mean particle volume has recently been treated in Cabo et al. (2003). It is here shown that an estimator based on planar observation is one order of magnitude more efficient than the traditional one based on observation along lines. In the present paper, we concentrate on the ordinary (unweighted) particle volume distribution. It is shown that an estimator of mean particle volume based on planar observations is superior to one based on line observations, especially for elongated particles. The variance of the estimator can be decomposed into the variance due to the stereological estimation procedure and the variance due to the variability in the particle volumes. We will show how to estimate these variance components separately, from sectional data. If the variance due to the stereological estimation procedure is small compared to the variance due to the variability in the particle volumes, the distribution of estimated particle volumes can be regarded as an estimate of the distribution of the true particle volumes.

\section{MARKED POINT PROCESSES}

We define the particle model by means of marked point processes. For more details, we refer to Stoyan et al. (1995). Let $\Psi_{m}=\left\{\left[X_{i} ; \Xi_{i}\right]\right\}$ be a marked point process such that $X_{i}$ is a point in $\mathbb{R}^{n}$ and $\Xi_{i}$ belongs to the space $\mathscr{M}_{d}$ of $d$-dimensional differentiable manifolds in $\mathbb{R}^{n}$ with finite $d$-dimensional Hausdorff measure and with the reference point at the origin $O$. The point $X_{i}$ then serves as a reference point of $X_{i}+\Xi_{i}$, the $i$-th particle.

The marked point process $\Psi_{m}$ will be assumed to be stationary, i.e., $\Psi_{m}+x=\left\{\left[X_{i}+x ; \Xi_{i}\right]\right\}$ has the same distribution as $\Psi_{m}$ for every $x \in \mathbb{R}^{n}$. Stationarity of $\Psi_{m}$ implies stationarity of the unmarked point process $\Psi=\left\{X_{i}\right\}$. Denote by $\lambda$ its intensity and assume that $0<\lambda<\infty$. Let $S O\left(n, L_{r}\right)$ be the subgroup of $S O(n)$ consisting of rotations keeping an $r$-dimensional linear subspace $L_{r}$ fixed. Then, $\Psi_{m}$ is said to be invariant under rotations in $S O\left(n, L_{r}\right)$ if $B \Psi_{m}=\left\{\left[B X_{i} ; B \Xi_{i}\right]\right\}$ and $\Psi_{m}$ have the same distribution for all rotations $B \in S O\left(n, L_{r}\right)$.

The intensity measure of the marked point process is defined for $A \in \mathscr{B}\left(\mathbb{R}^{n}\right)$ and $U \in \mathscr{B}\left(\mathscr{M}_{d}\right)$ as

$$
\Lambda_{m}(A \times U)=\mathrm{E} \sum_{i} \mathbf{1}_{A}\left(X_{i}\right) \mathbf{1}_{U}\left(\Xi_{i}\right)
$$

where $\mathbf{1}_{A}(\cdot)$ stands for the indicator function of the set $A$ and $\mathscr{B}$ denotes the Borel $\sigma$-algebra. The stationarity 
of $\Psi_{m}$ implies the following decomposition

$$
\Lambda_{m}(A \times U)=\lambda V(A) P_{m}(U)
$$

where $V=\lambda_{n}$ is the volume and $P_{m}$ is the mark distribution. By $\Xi_{0}$ we denote a random manifold with distribution $P_{m}$. If $\Psi_{m}$ is invariant under rotations in $S O\left(n, L_{r}\right)$, then $B \Xi_{0}$ has the same distribution as $\Xi_{0}$ for all $B \in S O\left(n, L_{r}\right)$.

\section{THE LOCAL STEREOLOGICAL ESTIMATORS}

The local stereological estimators are based on information collected from section planes in $\mathbb{R}^{n}$ through a reference point of the particle. In this section, we present the actual form of local stereological estimators of Hausdorff measure for a generic particle $K \in \mathscr{M}_{d}$. Then a section plane of dimension $p$ is a $p$-dimensional linear subspace (for brevity called $p$ subspace) of $\mathbb{R}^{n}, p=0,1, \ldots, n$. For comprehensive exposition of local stereology, see Jensen (1998).

There are various forms of the local estimators, depending on the restriction put on the $p$-subspace. Denote by $\mathscr{L}_{p, L_{r}}^{n}$ the set of $p$-subspaces containing a fixed $r$-subspace $L_{r}, 0 \leq r<p \leq n$. Let $\lambda_{n}^{d}$ be the $d$ dimensional Hausdorff measure in $\mathbb{R}^{n}$ and let us use the short notation $\mathrm{d} x^{d}$ instead of $\lambda_{n}^{d}(\mathrm{~d} x)$. Note that the ordinary Lebesgue measure is $\lambda_{n}^{n}=\lambda_{n}$. For $K \in \mathscr{M}_{d}$, the local stereological estimator of $\lambda_{n}^{d}(K)$, based on a $p$-subspace $L_{p} \in \mathscr{L}_{p, L_{r}}^{n}, d-n+p \geq 0$, has the form, $c f$. Jensen (1998), (5.24),

$$
\begin{aligned}
m_{p, L_{r}}^{(n, d)}\left(K, L_{p}\right)= & \frac{\sigma_{n-r}}{\sigma_{p-r}} \int_{K \cap L_{p}}\left\|\pi_{L_{r}^{\perp}} x\right\|^{n-p} \\
& \times G\left(\operatorname{Tan}[K, x], L_{p}\right)^{-1} \mathrm{~d} x^{d-n+p},
\end{aligned}
$$

where $\sigma_{n}=2 \pi^{n / 2} / \Gamma(n / 2)$ is the surface area of the unit sphere $\mathbb{S}^{n-1}$ in $\mathbb{R}^{n}, \pi_{L_{r}^{\perp}}$ is the orthogonal projection onto $L_{r}^{\perp}$ and $G\left(\operatorname{Tan}[K, x], L_{p}\right)$ is the socalled $G$-factor defined in Jensen (1998), Definition 2.9. Note that in the case $d=n, G\left(\operatorname{Tan}[K, x], L_{p}\right)=1$ for arbitrary $L_{p}$.

In a design-based setting, Eq. 1 is an unbiased estimator of $\lambda_{n}^{d}(K)$. Thus, let $\mu_{p, L_{r}}^{n}$ be the unique probability measure on $\mathscr{L}_{p, L_{r}}^{n}$, invariant under rotations from $S O\left(n, L_{r}\right)$. In what follows, we will write $\mathrm{d} L_{p, L_{r}}^{n}$ as short for $\mu_{p, L_{r}}^{n}\left(\mathrm{~d} L_{p}\right)$. By an isotropic $p$-subspace in $\mathbb{R}^{n}$, containing the fixed $r$-subspace $L_{r}$, we mean a random $p$-subspace with constant density with respect to $\mu_{p, L_{r}}^{n}$. Then, if $K$ satisfies the regularity conditions stated in Jensen (1998), Proposition 5.4, and $\tilde{L}_{p}$ is an isotropic $p$-subspace containing $L_{r}$, the local estimator $m_{p, L_{r}}^{(n, d)}\left(K, \tilde{L}_{p}\right)$ is an unbiased estimator of $\lambda_{n}^{d}(K)$, i.e.,

$$
\lambda_{n}^{d}(K)=\int_{\mathscr{L}_{p, L_{r}}^{n}} m_{p, L_{r}}^{(n, d)}\left(K, L_{p}\right) \mathrm{d} L_{p, L_{r}}^{n}
$$

Example 1. For $K \in \mathscr{M}_{3}$ in $\mathbb{R}^{3}$ there are three local stereological estimators of the volume $V(K)$,

$$
\begin{aligned}
& m_{2, O}^{(3,3)}\left(K, L_{2}\right)=2 \int_{K \cap L_{2}}\|x\| \mathrm{d} x^{2}, \\
& m_{1, O}^{(3,3)}\left(K, L_{1}\right)=2 \pi \int_{K \cap L_{1}}\|x\|^{2} \mathrm{~d} x^{1}, \\
& m_{2, L_{1}}^{(3,3)}\left(K, L_{2}\right)=\pi \int_{K \cap L_{2}}\left\|\pi_{L_{1}^{\perp}} x\right\| \mathrm{d} x^{2} .
\end{aligned}
$$

The estimators Eq. 3 and Eq. 4 are related by a socalled Rao-Blackwell procedure. We have

$$
m_{2, O}^{(3,3)}\left(K, L_{2}\right)=\mathrm{E}\left[m_{1, O}^{(3,3)}\left(K, \tilde{L}_{1}\right) \mid L_{2}\right]
$$

if $\tilde{L}_{1}$ is an isotropic line in $L_{2}$.

For later reference, we also present the local estimator of $\lambda_{n}^{d}(K)^{2}$,

$$
\begin{aligned}
& \tilde{m}_{p, L_{r}}^{(n, d)}\left(K, L_{p}\right)=\frac{\sigma_{n-r} \sigma_{n-r-1}}{\sigma_{p-r} \sigma_{p-r-1}} \\
& \times \int_{K \cap L_{p}} \int_{K \cap L_{p}} \nabla_{r+2}\left(e_{1}, \ldots, e_{r}, x_{1}, x_{2}\right)^{n-p} \\
& \quad \times \prod_{i=1}^{2} G\left(\operatorname{Tan}\left[K, x_{i}\right], L_{p}\right)^{-1} \prod_{i=1}^{2} \mathrm{~d} x_{i}^{d-n+p},
\end{aligned}
$$

where $1 \leq r+1<p \leq n, d-n+p \geq 0$ and $\nabla_{q}\left(y_{1}, \ldots, y_{q}\right)$ denotes the $q$-dimensional Hausdorff measure of the parallelepiped spanned by $y_{1}, \ldots, y_{q}$. Assuming that $K$ satisfies the regularity conditions from Jensen (1998), Theorem 5.6, $\tilde{m}_{p, L_{r}}^{(n, d)}\left(K, \tilde{L}_{p}\right)$ is an unbiased estimator of $\lambda_{n}^{d}(K)^{2}$ if $\tilde{L}_{p}$ is an isotropic $p$ subspace, containing $L_{r}$.

Example 2. For $d=n=3, p=2$ and $r=0$, the estimator of $V(K)^{2}$ has the form

$$
\tilde{m}_{2, O}^{(3,3)}\left(K, L_{2}\right)=2 \pi \int_{K \cap L_{2}} \int_{K \cap L_{2}} \nabla_{2}(x, y) \mathrm{d} y^{2} \mathrm{~d} x^{2}
$$

and $\nabla_{2}(x, y)$ is twice the area of the triangle with vertices $O, x$ and $y$. 


\section{THE VARIANCE OF LOCAL ESTIMATORS}

We will now return to the model-based case. We let $\Xi_{0}$ be a generic random particle, distributed according to $P_{m}$ and denote by $\mathrm{E}_{m}$ the expectation with respect to this distribution. Let $L_{p(0)}$ be a fixed $p$-subspace in $\mathbb{R}^{n}$, containing an $r$-subspace $L_{r}, 0 \leq r<p \leq n$, $d-n+p \geq 0$.

Below, we give explicit results for the second moment of $m_{p, L_{r}}^{(n, d)}\left(\Xi_{0}, L_{p(0)}\right)$. For this purpose, the following proposition is very useful.

Proposition 1. Let $P_{m}$ be invariant under rotations in $S O\left(n, L_{r}\right)$. The estimator $m_{p, L_{r}}^{(n, d)}\left(\Xi_{0}, L_{p(0)}\right)$ has the same distribution as $m_{p, L_{r}}^{(n, d)}\left(\Xi_{0}, \tilde{L}_{p}\right)$, where $L_{p(0)} \in$ $\mathscr{L}_{p, L_{r}}^{n}$ is fixed, $\tilde{L}_{p}$ is an isotropic p-subspace containing $L_{r}$ and $\Xi_{0}$ and $\tilde{L}_{p}$ are independent.

Proof. The result follows from the fact that for any non-negative measurable function $h$,

$$
\begin{array}{rl}
\mathrm{E}_{m} & h\left(m_{p, L_{r}}^{(n, d)}\left(\Xi_{0}, L_{p(0)}\right)\right) \\
= & \mathrm{E}_{m} \int_{\mathscr{L}_{p, L_{r}}^{n}} h\left(m_{p, L_{r}}^{(n, d)}\left(\Xi_{0}, L_{p}\right)\right) \mathrm{d} L_{p, L_{r}}^{n} .
\end{array}
$$

The left-hand side can be rewritten using the invariance of $P_{m}$ under rotations in $S O\left(n, L_{r}\right)$ and Jensen (1998), Lemma 8.4,

$$
\begin{array}{rl}
\mathrm{E}_{m} & h\left(m_{p, L_{r}}^{(n, d)}\left(\Xi_{0}, L_{p(0)}\right)\right) \\
= & \mathrm{E}_{m} h\left(m_{p, L_{r}}^{(n, d)}\left(B^{T} \Xi_{0}, L_{p(0)}\right)\right) \\
= & \mathrm{E}_{m} h\left(m_{p, L_{r}}^{(n, d)}\left(\Xi_{0}, B L_{p(0)}\right)\right),
\end{array}
$$

where $B \in S O\left(n, L_{r}\right)$. From invariant measure theory there exists an invariant probability measure $\alpha_{(r)}^{n}$ on $S O\left(n, L_{r}\right)$. Note that $\mathscr{L}_{p, L_{r}}^{n}=\left\{B L_{p(0)}: B \in S O\left(n, L_{r}\right)\right\}$ and

$$
\int_{S O\left(n, L_{r}\right)} g\left(B L_{p(0)}\right) \alpha_{(r)}^{n}(\mathrm{~d} B)=\int_{\mathscr{L}_{p, L_{r}}^{n}} g\left(L_{p}\right) \mathrm{d} L_{p, L_{r}}^{n}
$$

for any non-negative measurable function $g$ on $\mathscr{L}_{p, L_{r}}^{n}$. Thus,

$$
\begin{array}{rl}
\mathrm{E}_{m} & h\left(m_{p, L_{r}}^{(n, d)}\left(\Xi_{0}, L_{p(0)}\right)\right) \\
= & \int_{S O\left(n, L_{r}\right)} \mathrm{E}_{m} h\left(m_{p, L_{r}}^{(n, d)}\left(\Xi_{0}, B L_{p(0)}\right)\right) \alpha_{(r)}^{n}(\mathrm{~d} B) \\
= & \mathrm{E}_{m} \int_{S O\left(n, L_{r}\right)} h\left(m_{p, L_{r}}^{(n, d)}\left(\Xi_{0}, B L_{p(0)}\right)\right) \alpha_{(r)}^{n}(\mathrm{~d} B) \\
= & \mathrm{E}_{m} \int_{\mathscr{L}_{p, L_{r}}^{n}} h\left(m_{p, L_{r}}^{(n, d)}\left(\Xi_{0}, L_{p}\right)\right) \mathrm{d} L_{p, L_{r}}^{n} .
\end{array}
$$

When convenient we use the short notation $m\left(\Xi_{0}\right)$ for $m_{p, L_{r}}^{(n, d)}\left(\Xi_{0}, L_{p(0)}\right)$ and $m\left(\Xi_{0}, \tilde{L}_{p}\right)$ for $m_{p, L_{r}}^{(n, d)}\left(\Xi_{0}, \tilde{L}_{p}\right)$. Using Proposition 1 and Eq. 2, we get

$$
\mathrm{E}_{m} m\left(\Xi_{0}\right)=\mathrm{E} m\left(\Xi_{0}, \tilde{L}_{p}\right)=\mathrm{E}_{m} \lambda_{n}^{d}\left(\Xi_{0}\right) .
$$

Moreover, the relation Eq. 2 for a fixed $\Xi_{0}$ can be written as

$$
\mathrm{E}\left[m\left(\Xi_{0}, \tilde{L}_{p}\right) \mid \Xi_{0}\right]=\lambda_{n}^{d}\left(\Xi_{0}\right)
$$

almost surely, and for the variance of $m\left(\Xi_{0}\right)$, we get

$$
\begin{aligned}
& \operatorname{var}_{m} m\left(\Xi_{0}\right)=\operatorname{var} m\left(\Xi_{0}, \tilde{L}_{p}\right) \\
& =\mathrm{E}_{m} \operatorname{var}\left[m\left(\Xi_{0}, \tilde{L}_{p}\right) \mid \Xi_{0}\right]+\operatorname{var}_{m} \mathrm{E}\left[m\left(\Xi_{0}, \tilde{L}_{p}\right) \mid \Xi_{0}\right] \\
& =\mathrm{E}_{m} \operatorname{var}\left[m\left(\Xi_{0}, \tilde{L}_{p}\right) \mid \Xi_{0}\right]+\operatorname{var}_{m} \lambda_{n}^{d}\left(\Xi_{0}\right) \\
& \geq \operatorname{var}_{m} \lambda_{n}^{d}\left(\Xi_{0}\right) .
\end{aligned}
$$

Hence,

$$
\operatorname{var}_{m} \lambda_{n}^{d}\left(\Xi_{0}\right) \leq \operatorname{var}_{m} m\left(\Xi_{0}\right) .
$$

Generally, two random variables with the same expectation and variance don't have to be equal almost surely. But in our situation we can show that the equality of variances suffices.

Proposition 2. Let $\Xi_{0}$ be a typical manifold with distribution $P_{m}$ which is invariant under rotations in $S O\left(n, L_{r}\right)$. Then $\operatorname{var}_{m} \lambda_{n}^{d}\left(\Xi_{0}\right)=\operatorname{var}_{m} m\left(\Xi_{0}\right)$ if and only if $m\left(\Xi_{0}\right)=\lambda_{n}^{d}\left(\Xi_{0}\right)$ almost surely.

Proof. The equality in Eq. 9 happens if

$$
\mathrm{E}_{m} \operatorname{var}\left[m\left(\Xi_{0}, \tilde{L}_{p}\right) \mid \Xi_{0}\right]=0
$$

which can be rewritten (using the independence of $\Xi_{0}$ and $\left.\tilde{L}_{p}\right)$ as

$\int_{\mathscr{M}_{d}} \int_{\mathscr{L}_{p, L_{r}}^{n}}\left(m\left(K_{0}, L_{p}\right)-\lambda_{n}^{d}\left(K_{0}\right)\right)^{2} \mathrm{~d} L_{p, L_{r}}^{n} P_{m}\left(\mathrm{~d} K_{0}\right)=0$.

This in turn implies that $m\left(K_{0}, L_{p}\right)=\lambda_{n}^{d}\left(K_{0}\right)$ for $\left(P_{m} \times\right.$ $\left.\mu_{p, L_{r}}^{n}\right)$-a.a. $\left(K_{0}, L_{p}\right)$. It can be deduced that for $\mu_{p, L_{r}}^{n}{ }^{-}$ a.a. $L_{p(0)}$ we have $m\left(K_{0}\right)=\lambda_{n}^{d}\left(K_{0}\right)$ for $P_{m}$-a.a. $K_{0} \in$ $\mathscr{M}_{d}$. We would like to show this for all $p$-subspaces $L_{p(0)}$.

Let us suppose that there exists a subspace $L_{p(0)}$ and a set $A\left(L_{p(0)}\right) \in \mathscr{B}\left(\mathscr{M}_{d}\right)$ with $P_{m}\left(A\left(L_{p(0)}\right)\right)>0$ such that $m\left(K_{0}, L_{p(0)}\right) \neq \lambda_{n}^{d}\left(K_{0}\right)$ for $K_{0} \in A\left(L_{p(0)}\right)$. For any $L_{p} \in \mathscr{L}_{p, L_{r}}^{n}$, we can find $B \in S O\left(n, L_{r}\right)$ such that $L_{p}=B L_{p(0)}$. Since $m\left(K_{0}, L_{p(0)}\right)=$ 
$m\left(B K_{0}, B L_{p(0)}\right)$, see Jensen (1998), Lemma 8.4, we have $m\left(B K_{0}, L_{p}\right) \neq \lambda_{n}^{d}\left(K_{0}\right)$ for $K_{0} \in A\left(L_{p(0)}\right)$. Therefore, $m\left(K_{0}, L_{p}\right) \neq \lambda_{n}^{d}\left(K_{0}\right)$ on the set $A=$ $\left\{\left(K_{0}, L_{p}\right): K_{0} \in A\left(L_{p}\right), L_{p} \in \mathscr{L}_{p, L_{r}}^{n}\right\}$, where $A\left(L_{p}\right)=$ $B^{-1} A\left(L_{p(0)}\right)$. But from the invariance of $P_{m}$ under rotations in $S O\left(n, L_{r}\right)$ we obtain $P_{m}\left(A\left(L_{p}\right)\right)=$ $P_{m}\left(A\left(L_{p(0)}\right)\right)>0$ which means that $\left(P_{m} \times \mu_{p, L_{r}}^{n}\right)(A)>$ 0 and this leads us to a contradiction.

If $\operatorname{var}_{m} m\left(\Xi_{0}\right)=\operatorname{var}_{m} \lambda_{n}^{d}\left(\Xi_{0}\right)$, then the Hausdorff measure of the manifold is determined from the local section without error. Such local stereological estimators are exact, i.e., the variance of the estimator is created only by the randomness of particles. The simplest example of a particle with exact local volume estimator is a ball.

Proposition 3. Let $\Xi_{0}$ be an $n$-dimensional ball in $\mathbb{R}^{n}$ centred at $O$ with probability one. Then

$$
\operatorname{var}_{m} m_{p, L_{r}}^{(n, n)}\left(\Xi_{0}, L_{p(0)}\right)=\operatorname{var}_{m} V\left(\Xi_{0}\right)
$$

Proof. If $K=b_{n}(O, R)$ is an $n$-dimensional ball of radius $R$ with centre at the origin, then for all $L_{p} \in$ $\mathscr{L}_{p, L_{r}}^{n}$

$$
\begin{aligned}
\int_{K \cap L_{p}}\left\|\pi_{L_{r}^{\perp}} x\right\|^{n-p} \mathrm{~d} x^{p} \\
=\int_{\left\{x_{1}^{2}+\cdots+x_{p}^{2} \leq R^{2}\right\}}\left(x_{r+1}^{2}+\cdots+x_{p}^{2}\right)^{\frac{n-p}{2}} \mathrm{~d} x_{p} \cdots \mathrm{d} x_{1} .
\end{aligned}
$$

Since the right-hand side is $\omega_{n} R^{n} \frac{\sigma_{p-r}}{\sigma_{n-r}}$, where

$$
\omega_{n}=\lambda_{n}\left(b_{n}(O, 1)\right)=\frac{\pi^{\frac{n}{2}}}{\Gamma\left(\frac{n}{2}+1\right)}
$$

is the volume of the unit ball in $\mathbb{R}^{n}$, it follows that $m\left(K, L_{p}\right)=\lambda_{n}(K)$. Applying Proposition 1, Eq. 10 follows immediately.

In Jensen et al. (1999) the class of particles having an exact volume estimator (called quasi-spherical bodies) is studied.

By the similar reasoning as in the previous proof we can show that a sphere has exact surface area estimator.

Proposition 4. Let $\Xi_{0}$ be an $(n-1)$-dimensional sphere in $\mathbb{R}^{n}$ with centre $O$ almost surely. Then

$$
\operatorname{var}_{m} m_{p, L_{r}}^{(n, n-1)}\left(\Xi_{0}, L_{p(0)}\right)=\operatorname{var}_{m} \lambda_{n}^{n-1}\left(\Xi_{0}\right)
$$

Proof. It can be shown that $G\left(\operatorname{Tan}[K, x], L_{p}\right)=1$ if $K$ is an $(n-1)$-dimensional sphere in $\mathbb{R}^{n}$ and $x \in$ $K \cap L_{p}$. Now the proof proceeds along the same lines as the proof of Proposition 3, the integration over a $p$ dimensional ball is replaced by the integration over a $(p-1)$-dimensional sphere.

Remark 1. The lower dimensional spheres are not necessarily quasi-spherical. For example, consider the case $n=3, d=1, p=2$ and $r=0$. Then the local estimator of $\lambda_{3}^{1}\left(\Xi_{0}\right)$ has the form

$$
m_{2, O}^{(3,1)}\left(\Xi_{0}, L_{2(0)}\right)=\frac{4 R}{\sin \alpha}
$$

where $R$ is the radius of $\Xi_{0}$ and $\alpha$ is the angle between $L_{2(0)}$ and the plane containing the circle $\Xi_{0}$.

The local estimator Eq. 1 can be simplified if $K$ is star-shaped at $O$, i.e., $K \cap L_{1}$ is a line-segment for all $L_{1} \in \mathscr{L}_{1}^{n}$. Let $\rho_{K}(\omega)$ denote the radial function of $K$,

$$
\rho_{K}(\omega)=\max \{c: c \omega \in K\}, \quad \omega \in \mathbb{S}^{n-1},
$$

cf. Gardner (1995), p. 18. Let for $\omega \in \mathbb{S}^{n-1}$,

$$
\rho_{n, K}(\omega)= \begin{cases}\rho_{K}(\omega)^{n}+\rho_{K}(-\omega)^{n} & \text { for } O \in K, \\ \left.|| \rho_{K}(\omega)\right|^{n}-\left|\rho_{K}(-\omega)\right|^{n} \mid & \text { for } O \notin K\end{cases}
$$

be the $n$-chord function of the set $K$ at $O, c f$. Gardner (1995), Definition 6.1.1. Furthermore, let

$$
\tilde{\rho}_{n, K}\left(L_{p}\right)=\frac{1}{2 p} \int_{\mathbb{S}^{n-1} \cap L_{p}} \rho_{n, K}(\omega) \mathrm{d} \omega^{p-1}
$$

be the section function, $c f$. Gardner (1995), Chapter 7.

Proposition 5. Let $K$ be a star-shaped set at $O$. Then

$$
\begin{aligned}
& m_{p, L_{r}}^{(n, n)}\left(K, L_{p}\right)= \\
& \quad=\frac{\sigma_{n-r}}{\sigma_{p-r}} \frac{1}{2 n} \int_{\mathbb{S}^{n-1} \cap L_{p}} \rho_{n, K}(\omega)\left\|\pi_{L_{r}^{\perp}} \omega\right\|^{n-p} \mathrm{~d} \omega^{p-1} .
\end{aligned}
$$

Proof. Using the polar decomposition of Lebesgue measure we obtain

$$
\begin{aligned}
& \int_{K \cap L_{p}}\left\|\pi_{L_{r}^{\perp}} x\right\|^{n-p} \mathrm{~d} x^{p} \\
& =\frac{1}{2} \int_{\mathbb{S}^{n-1} \cap L_{p}} \int_{K \cap \operatorname{span}\{\omega\}}\|x\|^{n-1}\left\|\pi_{L_{r}^{\perp}} \omega\right\|^{n-p} \mathrm{~d} x^{1} \mathrm{~d} \omega^{p-1} \\
& =\frac{1}{2 n} \int_{\mathbb{S}^{n-1} \cap L_{p}} \rho_{n, K}(\omega)\left\|\pi_{L_{r}^{\perp}} \omega\right\|^{n-p} \mathrm{~d} \omega^{p-1}
\end{aligned}
$$


In particular, for $r=0$, the local stereological estimator is proportional to the section function

$$
m_{p, O}^{(n, n)}\left(K, L_{p}\right)=\frac{\omega_{n}}{\omega_{p}} \tilde{\rho}_{n, K}\left(L_{p}\right) .
$$

Our aim is now to derive some explicit results for the second moment of $m\left(\Xi_{0}\right)$. This will give an easy way of finding $\operatorname{var}_{m} m\left(\Xi_{0}\right)$ (without simulation) for particular choices of shapes of $\Xi_{0}$ and will give insight into what kind of shapes of $\Xi_{0}$ result in an estimator with large variance. In what follows we always assume that $\Xi_{0}$ is invariant under rotations in $S O\left(n, L_{r}\right)$.

Proposition 6. Let $\Xi_{0}$ be a symmetric and star-shaped set at $O$. Then for the local estimator with $d=n, p=1$ and $r=0$ we have

$$
\mathrm{E}_{m} m_{1, O}^{(n, n)}\left(\Xi_{0}, L_{1(0)}\right)^{2}=\omega_{n}^{2} \mathrm{E}_{m} \int_{\mathbb{S}^{n-1}} \rho_{\Xi_{0}}(\omega)^{2 n} \frac{\mathrm{d} \omega^{n-1}}{\sigma_{n}} .
$$

Proof. Since $\Xi_{0}$ is star-shaped at $O$, we see from Eq. 11 that the local estimator is proportional to the $n$-chord function,

$$
m_{1, O}^{(n, n)}\left(\Xi_{0}, \operatorname{span}\{\omega\}\right)=\frac{\omega_{n}}{2} \rho_{n, \Xi_{0}}(\omega), \quad \omega \in \mathbb{S}^{n-1} .
$$

Using the symmetry of $\Xi_{0}\left(\rho_{\Xi_{0}}(\omega)=\rho_{\Xi_{0}}(-\omega)\right)$ and Proposition 1 we obtain the stated result.

Sometimes, it can be useful to have an alternative expression of $\mathrm{E}_{m} m_{1, O}^{(n, n)}\left(\Xi_{0}, L_{1(0)}\right)^{2}$.

Proposition 7. Let $\Xi_{0}$ be symmetric and star-shaped set at $O$. Then for the local estimator with $d=n, p=1$ and $r=0$ we have

$$
\mathrm{E}_{m} m_{1, O}^{(n, n)}\left(\Xi_{0}, L_{1(0)}\right)^{2}=2 \omega_{n} \mathrm{E}_{m} \int_{\Xi_{0}}\|x\|^{n} \mathrm{~d} x^{n} .
$$

Proof. The formula in Proposition 6 can be rewritten as

$$
\begin{array}{rl}
\mathrm{E}_{m} & m\left(\Xi_{0}\right)^{2} \\
= & \omega_{n}^{2} \mathrm{E}_{m} \int_{\mathbb{S}^{n-1}} \int_{\Xi_{0} \cap \operatorname{span}\{\omega\}} n\|x\|^{2 n-1} \mathrm{~d} x^{1} \frac{\mathrm{d} \omega^{n-1}}{\sigma_{n}} \\
= & n \omega_{n}^{2} \mathrm{E}_{m} \int_{\mathscr{L}_{1, O}^{n}} \int_{\Xi_{0} \cap L_{1}}\|x\|^{2 n-1} \mathrm{~d} x^{1} \mathrm{~d} L_{1, O}^{n} \\
= & 2 \omega_{n} \mathrm{E}_{m} \int_{\Xi_{0}}\|x\|^{n} \mathrm{~d} x^{n},
\end{array}
$$

where in the last step we have used (Jensen, 1998), Proposition 4.1 with $g(x)=\|x\|^{n}$.
Remark 2. The assumptions of the previous two propositions are not restrictive as they may appear. If $\Xi_{0}$ is not a symmetric and star-shaped set, we can define an equivalent symmetric star-shaped set $\operatorname{star}\left(\Xi_{0}\right), c f$.Jensen (2000), by

$$
\rho_{\operatorname{star}\left(\Xi_{0}\right)}(\omega)=\omega_{n}^{-1 / n} m_{1, O}^{(n, n)}\left(\Xi_{0}, \operatorname{span}\{\omega\}\right)^{1 / n},
$$

$\omega \in \mathbb{S}^{n-1}$. Obviously, $m\left(\Xi_{0}\right)=m\left(\operatorname{star}\left(\Xi_{0}\right)\right)$, thus Proposition 6 and Proposition 7 can be used for any $\Xi_{0}$ if $\Xi_{0}$ is replaced by $\operatorname{star}\left(\Xi_{0}\right)$ in the right-hand side of Propositions 6 and 7.

Now we turn to the case $p \geq r+2$.

Proposition 8. For $p \geq r+2$ the second moment of the local estimator is

$$
\begin{gathered}
\mathrm{E}_{m} m_{p, L_{r}}^{(n, d)}\left(\Xi_{0}, L_{p(0)}\right)^{2}=\frac{\sigma_{n-r} \sigma_{p-r-1}}{\sigma_{n-r-1} \sigma_{p-r}} \mathrm{E}_{m} \int_{\Xi_{0}} \int_{\Xi_{0}} \\
\left(1-\left\langle\frac{\pi_{L_{r}^{\perp}} x}{\left\|\pi_{L_{r}} x\right\|}, \frac{\pi_{L_{r}^{\perp}} y}{\left\|\pi_{L_{r}} y\right\|}\right\rangle^{2}\right)^{-\frac{n-p}{2}} \mathrm{~d} x^{d} \mathrm{~d} y^{d} .
\end{gathered}
$$

Proof. Under the regularity conditions of (Jensen, 1998), Theorem 5.6, we know that

$$
\begin{aligned}
& \mathrm{E}_{m} m\left(\Xi_{0}\right)^{2}=\frac{\sigma_{n-r}^{2}}{\sigma_{p-r}^{2}} \mathrm{E}_{m} \int_{\mathscr{L}_{p, L_{r}}^{n}} \int_{\Xi_{0} \cap L_{p}} \int_{\Xi_{0} \cap L_{p}}\left\|\pi_{L_{r}^{\perp}} x\right\|^{n-p} \\
& \times G\left(\operatorname{Tan}\left[\Xi_{0}, x\right], L_{p}\right)^{-1}\left\|\pi_{L_{r}^{\perp}} y\right\|^{n-p} G\left(\operatorname{Tan}\left[\Xi_{0}, y\right], L_{p}\right)^{-1} \\
& \mathrm{~d} x^{d-n+p} \mathrm{~d} y^{d-n+p} \mathrm{~d} L_{p, L_{r}}^{n} .
\end{aligned}
$$

Using the generalized Blaschke-Petkantschin formula (Jensen, 1998), Theorem 5.6, with

$$
g(x, y)=\frac{\left\|\pi_{L_{r}^{\perp}} x\right\|^{n-p}\left\|\pi_{L_{r}^{\perp}} y\right\|^{n-p}}{\nabla_{2}\left(\pi_{L_{r}^{\perp}} x, \pi_{L_{r}^{\perp}} y\right)^{n-p}}
$$

we get $(d-n+p \geq 0)$

$$
\begin{aligned}
& \mathrm{E}_{m} m_{p, L_{r}}^{(n, d)}\left(\Xi_{0}, L_{p(0)}\right)^{2}=\frac{\sigma_{n-r} \sigma_{p-r-1}}{\sigma_{n-r-1} \sigma_{p-r}} \\
& \quad \times \mathrm{E}_{m} \iint_{\Xi_{0}} \frac{\left\|\pi_{\Xi_{r}^{\perp}} x\right\|^{n-p}\left\|\pi_{L_{r}^{\perp}} y\right\|^{n-p}}{\nabla_{2}\left(\pi_{L_{r}^{\perp}} x, \pi_{L_{r}^{\perp}} y\right)^{n-p}} \mathrm{~d} x^{d} \mathrm{~d} y^{d} .
\end{aligned}
$$

Notice that due to the assumed regularity conditions, $\nabla_{2}\left(\pi_{L_{r}^{\perp}} x, \pi_{L_{r}^{\perp}} y\right)=0$ on a set of $\left(\lambda_{n}^{d} \times \lambda_{n}^{d}\right)$-measure zero and the integral on the right-hand side is well-defined. The result now follows immediately from $\nabla_{2}(x, y)=$ $\left(\|x\|^{2}\|y\|^{2}-\langle x, y\rangle^{2}\right)^{1 / 2}$. 
Note that the second moment of $m_{p, L_{r}}^{(n, d)}\left(\Xi_{0}, L_{p(0)}\right)$ does not depend on the $G$-factor. For $n$-dimensional particles the formula for the variance given in Proposition 7 was expressed through integrals over $\mathbb{S}^{n-1} \cap L_{r}^{\perp}$ in Jensen et al. (1999). Finally, we consider the special case of star-shaped particles and $r=0$.

Proposition 9. Let $\Xi_{0}$ be a star-shaped set at $O$ with $O \in \Xi_{0}$ almost surely. Suppose that $p \geq 2$. Then

$$
\begin{aligned}
& \mathrm{E}_{m} m_{p, O}^{(n, n)}\left(\Xi_{0}, L_{p(0)}\right)^{2}=\frac{\sigma_{n} \sigma_{p-1}}{4 n^{2} \sigma_{n-1} \sigma_{p}} \\
& \times \mathrm{E}_{m} \int_{\mathbb{S}^{n-1}} \int_{\mathbb{S}^{n-1}} \rho_{n, \Xi_{0}}\left(\omega_{1}\right) \rho_{n, \Xi_{0}}\left(\omega_{2}\right) \\
& \quad \times\left(1-\left\langle\omega_{1}, \omega_{2}\right\rangle^{2}\right)^{-\frac{n-p}{2}} \mathrm{~d} \omega_{1}^{n-1} \mathrm{~d} \omega_{2}^{n-1} .
\end{aligned}
$$

Proof. It is not difficult to see that the result follows immediately from Proposition 8 and the following formulation of polar decomposition of Lebesgue measure

$$
\begin{aligned}
\int_{K} g\left(\frac{x}{\|x\|}\right) \mathrm{d} x^{n} \\
\quad=\frac{1}{2} \int_{\mathbb{S}^{n-1}} \int_{\operatorname{span}\{\omega\}} \mathbf{1}_{K}(x) g(\omega)\|x\|^{n-1} \mathrm{~d} x^{1} \mathrm{~d} \omega^{n-1} .
\end{aligned}
$$

\section{EXAMPLES}

In this section we use the results to find explicit expressions of the variance of local stereological estimators for specific particle shapes.

\section{THE PLANAR CASE}

For $n=d=2, p=1$ and $r=0$, the variance can easily be determined, using Proposition 6 or Proposition 7 for various shapes of $\Xi_{0}$. We give the formulas for $\operatorname{var}_{m} m_{1, O}^{(2,2)}\left(\Xi_{0}, L_{1(0)}\right)$ for three particular choices of $\Xi_{0}$ centred at $O$ :

- rectangle with sides of lengths $a, b$

$$
\frac{\pi}{6} \mathrm{E} a b\left(a^{2}+b^{2}\right)-(\mathrm{E} a b)^{2}
$$

- ellipse with semiaxes of lengths $a, b$

$$
\frac{\pi^{2}}{2} \mathrm{E} a b\left(a^{2}+b^{2}\right)-\pi^{2}(\mathrm{E} a b)^{2}
$$

- equilateral triangle with the side of length $a$

$$
\frac{\pi \sqrt{3}(21-8 \log 2)}{432} \mathrm{E} a^{4}-\frac{3}{16}\left(\mathrm{E} a^{2}\right)^{2}
$$

\section{TRIAXIAL ELLIPSOIDS}

We suppose that $\Xi_{0}$ is an ellipsoid centred at $O$ and with semiaxes of lengths $a, b$ and $c$. In $\mathbb{R}^{3}$ there are three local stereological volume estimators, namely Eq. 3, Eq. 4 and Eq. 5.

For $p=1$ the second moment of $m_{1, O}^{(3,3)}\left(\Xi_{0}, L_{1(0)}\right)$ can be written as (using Proposition 6 and spherical coordinates)

$$
\begin{aligned}
\frac{8 \pi}{9} & \mathrm{E} \int_{0}^{\pi} \int_{-\frac{\pi}{2}}^{\frac{\pi}{2}}\left(\frac{\cos ^{2} \theta \cos ^{2} \varphi}{a^{2}}\right. \\
& \left.+\frac{\cos ^{2} \theta \sin ^{2} \varphi}{b^{2}}+\frac{\sin ^{2} \theta}{c^{2}}\right)^{-3} \cos \theta \mathrm{d} \theta \mathrm{d} \varphi
\end{aligned}
$$

or in the form

$$
\begin{aligned}
& \frac{8 \pi}{9} \mathrm{E} a b c \int_{0}^{\pi} \int_{-\frac{\pi}{2}}^{\frac{\pi}{2}}\left(a^{2} \cos ^{2} \theta \cos ^{2} \varphi\right. \\
& \left.\quad+b^{2} \cos ^{2} \theta \sin ^{2} \varphi+c^{2} \sin ^{2} \theta\right)^{3 / 2} \cos \theta \mathrm{d} \theta \mathrm{d} \varphi,
\end{aligned}
$$

where we used Proposition 7 and the transformation

$$
x=(\operatorname{arcos} \theta \cos \varphi, b r \cos \theta \sin \varphi, c r \sin \theta)^{T},
$$

$r \in(0,1), \theta \in\left(-\frac{\pi}{2}, \frac{\pi}{2}\right), \varphi \in(0,2 \pi)$. If we suppose that there exist constants $a_{0}, b_{0}, c_{0}$ and a non-negative random variable $\rho$ such that $a=\rho a_{0}, b=\rho b_{0}$ and $c=\rho c_{0}$ (i.e., the typical particle shape is fixed, only size and direction are random), then the variance of the local estimator becomes

$$
\operatorname{var}_{m} m\left(\Xi_{0}\right)^{2}=V_{0}^{2}\left(\kappa \mathrm{E} \rho^{6}-\left(\mathrm{E} \rho^{3}\right)^{2}\right)
$$

where $V_{0}=\frac{4 \pi}{3} a_{0} b_{0} c_{0}$ is the volume of an ellipsoid with semiaxes $a_{0}, b_{0}, c_{0}$ and the constant $\kappa$ can be determined from either Eq. 12 or Eq. 13 by means of numerical integration.

For $p=2$ and $r=0$ we can proceed in similar way. From Proposition 9 we have

$$
\begin{aligned}
& \mathrm{E}_{m} m_{2, O}^{(3,3)}\left(\Xi_{0}, L_{2(0)}\right)^{2}=\frac{2}{9 \pi} \mathrm{E}_{m} \int_{\mathbb{S}^{2} \mathbb{S}^{2}}\left(1-\left\langle\omega_{1}, \omega_{2}\right\rangle^{2}\right)^{-\frac{1}{2}} \\
& \times \rho_{\Xi_{0}}\left(\omega_{1}\right)^{3} \rho_{\Xi_{0}}\left(\omega_{2}\right)^{3} \mathrm{~d} \omega_{1}^{2} \mathrm{~d} \omega_{2}^{2} .
\end{aligned}
$$

Note that there is a mistake in Jensen (2000), the constant $\frac{8}{\pi^{3}}$ should be replaced by $\frac{1}{8 \pi^{3}}$. For fixed $\Xi_{0}$ the double integral can again be computed numerically. The formula Eq. 14 still holds, the values of $\kappa$ for several choices of ratios $a_{0} / b_{0}$ and $b_{0} / c_{0}$ are summarized in Table 1 . We have also computed $\kappa$ for 
an intermediate estimator, usually called the nucleator, cf. Gundersen (1988),

$$
\begin{aligned}
& \bar{m}_{1, O}^{(3,3)}\left(\Xi_{0}\right)= \\
& \quad \frac{1}{2}\left[m_{1, O}^{(3,3)}\left(\Xi_{0}, \operatorname{span}\left\{\omega_{1}\right\}\right)+m_{1, O}^{(3,3)}\left(\Xi_{0}, \operatorname{span}\left\{\omega_{2}\right\}\right)\right],
\end{aligned}
$$

where $\omega_{1} \in \mathbb{S}^{2} \cap L_{2}$ is an isotropic direction in an isotropic plane $L_{2}$ and $\omega_{2} \in \mathbb{S}^{2} \cap L_{2}$ is orthogonal to $\omega_{1}$. Our approach based on numerical integration enables more precise results than those obtained by simulation in Jensen (2000). Note that $\sqrt{\kappa-1}$ is the coefficient of error of the local volume estimator for a corresponding ellipsoid with semiaxes $a_{0}, b_{0}$ and $c_{0}$.

Table 1. The values of $\kappa$ from Eq. 14 for three types of volume estimators and various shapes of ellipsoids.

\begin{tabular}{cc|rrr}
$a_{0} / b_{0}$ & $b_{0} / c_{0}$ & $m_{1, O}\left(\Xi_{0}\right)$ & $\bar{m}_{1, O}\left(\Xi_{0}\right)$ & $m_{2, O}\left(\Xi_{0}\right)$ \\
\hline 1 & 1 & 1 & 1 & 1 \\
1 & 2 & 1.34440 & 1.10854 & 1.07945 \\
1 & 4 & 2.43264 & 1.58048 & 1.26608 \\
\hline 2 & 1 & 1.51757 & 1.16635 & 1.11835 \\
2 & 2 & 2.60921 & 1.63012 & 1.31307 \\
2 & 4 & 5.03481 & 2.79692 & 1.59320 \\
\hline 4 & 1 & 4.42495 & 2.45371 & 1.59821 \\
4 & 2 & 8.51728 & 4.44465 & 2.03585 \\
4 & 4 & 16.87915 & 8.60156 & 2.56130 \\
\hline
\end{tabular}

Higher values of $\kappa$ mean higher variance caused by the local stereological estimation. For ball $(\kappa=1)$ we have an exact estimator with

$$
\operatorname{var}_{m} m\left(\Xi_{0}\right)=V_{0}^{2} \operatorname{var} \rho^{3}=\operatorname{var}_{m} \lambda_{3}\left(\Xi_{0}\right) .
$$

Note that the error is larger for prolate spheroids $(b=$ $c)$ than for the corresponding oblate spheroids $(a=b)$. In view of Eq. 6, it is not surprising that smaller values of error are obtained for the local estimator based on plane sections.

In the remainder of this subsection we consider the last local volume estimator Eq. 5. Obviously, it depends on the choice of the fixed line $L_{1}$ (usually called vertical axis) relative to the ellipsoid. We assume that the vertical axis has the same direction as one of the semiaxes of the ellipsoid (say the one of length $c$ ). Then the profile $\Xi_{0} \cap L_{2(0)}$ is a planar ellipse with semiaxes of length $A$ and $c$. Hence, the local estimator has the following form

$$
\begin{aligned}
m_{2, L_{1}}^{(3,3)}\left(\Xi_{0}, L_{2(0)}\right) & =\pi \int_{0}^{2 \pi} \int_{0}^{1} A^{2} c r^{2}|\cos \varphi| \mathrm{d} r \mathrm{~d} \varphi \\
& =\frac{4 \pi}{3} A^{2} c
\end{aligned}
$$

Let $\alpha$ be the angle between $L_{2(0)}$ and the semiaxis of length $a$. Then $A$ can be expressed as the function of $a$, $b$ and $\alpha$ and the second moment of $m\left(\Xi_{0}\right)$ is

$$
\begin{aligned}
\mathrm{E} m_{2, L_{1}}^{(3,3)}\left(\Xi_{0}, L_{2(0)}\right)^{2} & =\frac{16 \pi}{9} \mathrm{E} \int_{0}^{\pi} \frac{c^{2}}{\left(\frac{\cos ^{2} \alpha}{a^{2}}+\frac{\sin ^{2} \alpha}{b^{2}}\right)^{2}} \mathrm{~d} \alpha \\
& =\frac{8 \pi^{2}}{9} \mathrm{E} a b c^{2}\left(a^{2}+b^{2}\right) .
\end{aligned}
$$

For fixed shape of $\Xi_{0}$ the constant $\kappa$ in Eq. 14 does not depend on $c_{0}$,

$$
\kappa=\frac{a_{0}^{2}+b_{0}^{2}}{2 a_{0} b_{0}} .
$$

For the values mentioned in Table 1 we get $\kappa=1$ if $a_{0} / b_{0}=1, \kappa=1.25$ if $a_{0} / b_{0}=2$ and $\kappa=2.125$ if $a_{0} / b_{0}=4$.

\section{OTHER SPATIAL PARTICLES}

A table similar to Table 1 can be determined for other choices of particle shape.

As an example, let $\Xi_{0}$ be obtained by scaling the prototype cuboid with edges of lenghts $a_{0}, b_{0}, c_{0}$. It means that $\Xi_{0}$ is an isotropically oriented cuboid with edges of lengths $\rho a_{0}, \rho b_{0}, \rho c_{0}$, where $\rho$ is a non-negative random variable. Then Eq. 14 holds with $V_{0}=a_{0} b_{0} c_{0}$ and $\kappa$ can be calculated numerically (see Table 2). The obtained values are slightly larger than for ellipsoids.

Table 2. The values of $\kappa$ for various shapes of cuboids.

\begin{tabular}{cc|rrr}
$a_{0} / b_{0}$ & $b_{0} / c_{0}$ & $m_{1, O}\left(\Xi_{0}\right)$ & $\bar{m}_{1, O}\left(\Xi_{0}\right)$ & $m_{2, O}\left(\Xi_{0}\right)$ \\
\hline 1 & 1 & 1.15338 & 1.09933 & 1.01883 \\
1 & 2 & 1.52818 & 1.19482 & 1.10735 \\
1 & 4 & 2.72926 & 1.73022 & 1.31183 \\
\hline 2 & 1 & 1.70178 & 1.21758 & 1.15148 \\
2 & 2 & 2.87074 & 1.71663 & 1.36691 \\
2 & 4 & 5.49895 & 2.99522 & 1.67056 \\
\hline 4 & 1 & 4.75547 & 2.57099 & 1.68498 \\
4 & 2 & 9.07151 & 4.68338 & 2.16437 \\
4 & 4 & 17.93156 & 9.09621 & 2.72906 \\
\hline
\end{tabular}

If the particle distribution is invariant under the rotations keeping the vertical axis fixed and the direction of the vertical axis is parallel to the edge of length $c$ of the cuboid, then

$$
\kappa=\frac{\pi}{6} \cdot \frac{a_{0}^{2}+b_{0}^{2}}{a_{0} b_{0}} .
$$

As the next example consider a regular tetrahedron of random size. For the estimator based on line section $\kappa=1.20049$ and for the estimator based on plane section $\kappa=1.01775$. 


\section{HIGHER DIMENSIONS}

If $n>3$ we do not always have to use numerical integration in order to derive the explicit formula for the variance. For instance, if $\Xi_{0}$ is an ellipsoid in $\mathbb{R}^{4}$ with semiaxes $a_{1}, a_{2}, a_{3}, a_{4}$, then

$$
\begin{aligned}
\mathrm{E}_{m} & m_{1, O}^{(4,4)}\left(\Xi_{0}, L_{1(0)}\right)^{2} \\
= & \frac{1}{8} \pi^{4} \mathrm{E} a_{1} a_{2} a_{3} a_{4}\left(\frac{1}{6} \sum_{i=1}^{4} a_{i}^{4}+\frac{1}{12}\left(\sum_{i=1}^{4} a_{i}^{2}\right)^{2}\right) .
\end{aligned}
$$

This result can be derived from Proposition 7, using elliptical coordinates and lengthy but straightforward calculations.

\section{ESTIMATION OF VARIANCES}

It is interesting to find the estimators of the variances $\sigma_{m}^{2}=\operatorname{var}_{m} m_{p, L_{r}}^{(n, d)}\left(\Xi_{0}, L_{p(0)}\right)$ and $\sigma_{\lambda}^{2}=$ $\operatorname{var}_{m} \lambda_{n}^{d}\left(\Xi_{0}\right)$, separately, from sectional data. First we introduce ratio-unbiased estimators of $\mu=\mathrm{E}_{m} \lambda_{n}^{d}\left(\Xi_{0}\right)$, $\alpha_{m}^{2}=\mathrm{E}_{m} m_{p, L_{r}}^{(n, d)}\left(\Xi_{0}, L_{p(0)}\right)^{2}$ and $\alpha_{\lambda}^{2}=\mathrm{E}_{m} \lambda_{n}^{d}\left(\Xi_{0}\right)^{2}$, respectively.

Let $W$ be a fixed bounded Borel set in $\mathbb{R}^{n}$ with positive volume. We consider a sample of particles $X_{i}+\Xi_{i}$ with $X_{i}$ in the sampling window $W$. Then the local estimator is determined from the central section $\left(X_{i}+\Xi_{i}\right) \cap\left(X_{i}+L_{p(0)}\right)$ for each sampled particle. In order to be in accordance with Eq. 1 we can think that the centred particle $\Xi_{i}$ is sectioned by a fixed $p$ subspace $L_{p(0)} \in \mathscr{L}_{p, L_{r}}^{n}$, i.e.,

$$
m_{p, L_{r}}^{(n, d)}\left(X_{i}+\Xi_{i}, X_{i}+L_{p(0)}\right)=m_{p, L_{r}}^{(n, d)}\left(\Xi_{i}, L_{p(0)}\right) .
$$

In what follows we assume that the mark distribution $P_{m}$ of a stationary marked point process $\Psi_{m}$ is invariant under rotations in $S O\left(n, L_{r}\right)$. It can be shown that (see Jensen (1998), Proposition 8.5)

$$
\widehat{\mu}=\sum_{i} \mathbf{1}_{W}\left(X_{i}\right) m_{p, L_{r}}^{(n, d)}\left(X_{i}+\Xi_{i}, X_{i}+L_{p(0)}\right) / \Psi(W)
$$

is a ratio-unbiased estimator of the mean particle Hausdorff measure

$$
\mathrm{E}_{m} \lambda_{n}^{d}\left(\Xi_{0}\right)=\int_{\mathscr{M}_{d}} \lambda_{n}^{d}(K) P_{m}(\mathrm{~d} K) .
$$

The proof is based on Campbell's formula for marked point processes (see Stoyan et al. (1995), Section 4.2) and Eq. 8.

Using Eq. 7, we can estimate the second moment in the mark distribution, i.e., $\mathrm{E}_{m} \lambda_{n}^{d}\left(\Xi_{0}\right)^{2}$. Let $\Xi_{0}$ satisfy the regularity conditions from Jensen (1998), Theorem 5.6, and let $L_{p(0)}$ be a fixed $p$-subspace in $\mathbb{R}^{n}$, containing $L_{r}, 1 \leq r+1<p \leq n, d-n+p \geq 0$. Proposition 8.7 in Jensen (1998) states that if $W$ is a bounded Borel set with positive volume, then

$$
\widehat{\alpha_{\lambda}^{2}}=\sum_{i} \mathbf{1}_{W}\left(X_{i}\right) \tilde{m}_{p, L_{r}}^{(n, d)}\left(X_{i}+\Xi_{i}, X_{i}+L_{p(0)}\right) / \Psi(W)
$$

is a ratio-unbiased estimator of $\mathrm{E}_{m} \lambda_{n}^{d}\left(\Xi_{0}\right)^{2}$.

We can also estimate $\mathrm{E}_{m} m_{p, L_{r}}^{(n, d)}\left(\Xi_{0}, L_{p(0)}\right)^{2}$ by

$$
\widehat{\alpha_{m}^{2}}=\sum_{i} \mathbf{1}_{W}\left(X_{i}\right) m_{p, L_{r}}^{(n, d)}\left(X_{i}+\Xi_{i}, X_{i}+L_{p(0)}\right)^{2} / \Psi(W) .
$$

This estimator is again ratio-unbiased, as can be easily seen from Campbell's formula for marked point processes.

The problem is how to estimate

$$
\mu^{2}=\left(\mathrm{E}_{m} m_{p, L_{r}}^{(n, d)}\left(\Xi_{0}, L_{p(0)}\right)\right)^{2}=\left(\mathrm{E}_{m} \lambda_{n}^{d}\left(\Xi_{0}\right)\right)^{2} .
$$

As long as we restrict ourselves to the case of independently marked point process (i.e., the $\Xi_{i}$ are independent and identically distributed and independent of $\Psi$ ),

$$
\widehat{\sigma_{m}^{2}}=\frac{1}{\Psi(W)-1} \sum_{i}\left(m\left(X_{i}+\Xi_{i}\right)-\widehat{\mu}\right)^{2}
$$

and

$$
\widehat{\sigma_{\lambda}^{2}}=\widehat{\alpha_{\lambda}^{2}}-(\widehat{\mu})^{2}+\frac{1}{\Psi(W)-1}\left(\widehat{\alpha_{m}^{2}}-(\widehat{\mu})^{2}\right)
$$

are unbiased estimators of $\sigma_{m}^{2}=\operatorname{var}_{m} m\left(\Xi_{0}\right)$ and $\sigma_{\lambda}^{2}=$ $\operatorname{var}_{m} \lambda_{n}^{d}\left(\Xi_{0}\right)$, respectively.

In the general case of dependent particles we propose following estimators of $\sigma_{m}^{2}$,

$$
\widehat{\sigma_{m}^{2}}=\frac{\sum_{h}\left(m\left(X_{i}+\Xi_{i}\right)-m\left(X_{j}+\Xi_{j}\right)\right)^{2}}{\sum_{h} 1}
$$

or the estimator taking into account edge corrections

$$
\widehat{\sigma_{m}^{2}}=\frac{\sum_{h} \frac{\left(m\left(X_{i}+\Xi_{i}\right)-m\left(X_{j}+\Xi_{j}\right)\right)^{2}}{V\left(\left(W-X_{i}\right) \cap\left(W-X_{j}\right)\right)}}{2 \sum_{h} \frac{1}{V\left(\left(W-X_{i}\right) \cap\left(W-X_{j}\right)\right)}},
$$

where $\sum_{h}$ means that the sum is taken over $i$ and $j$ such that $X_{i} \in W, X_{j} \in W$ and $\left\|X_{i}-X_{j}\right\| \geq h$. An appropriate choice of the parameter $h \geq 0$ is some trade-off between small value (larger bias) and 
large value (few observations, larger variance). Using Campbell's formula it is easy to show that $\widehat{\sigma_{m}^{2}}$ is in both cases ratio-unbiased if $m\left(X_{i}+\Xi_{i}\right)$ and $m\left(X_{j}+\Xi_{j}\right)$ are independent whenever $\left\|X_{i}-X_{j}\right\| \geq h_{0}$. The estimate of $\sigma_{\lambda}^{2}$ has then the form

$$
\widehat{\sigma_{\lambda}^{2}}=\widehat{\sigma_{m}^{2}}-\widehat{\alpha_{m}^{2}}+\widehat{\alpha_{V}^{2}}
$$

In applications, the distribution of estimated particle volumes (or other size parameters) has been used as an estimate of the true particle volume distribution. This procedure is justified if the variance due to the stereological estimation procedure is small compared to the variance due to the variability in the particle population. We can estimate both variances from central sections using Eq. 15 and Eq. 16 or Eq. 17, Eq. 18 and Eq. 19. If the estimates are closed we can expect that the distribution of estimated sizes will be close to the true size distribution. The practical implications of this observation will be investigated elsewhere.

\section{ACKNOWLEDGEMENTS}

This research has been supported by a Marie Curie Fellowship of the European Community Programme Improving the Human Research Potential and the Socioeconomic Knowledge Base under contract number HPMT-CT-2001-00364, the Danish Natural Science Research Council, the Grant Agency of the Czech Republic, project 201/06/0302 and the Czech Ministry of Education, project MSM 0021620839.

The work has been presented at the Conference $S^{4} \mathrm{G}$, Prague, June 26-29, 2006.

\section{REFERENCES}

Cabo A, Baddeley A (2003). Estimation of mean particle volume using the set covariance function. Adv Appl Prob 35:27-46.

Gardner RJ (1995). Geometric tomography. New York: Cambridge University Press.

Gundersen HJG (1988). The nucleator. J Microsc 143:3-45.

Jensen EBV (1998). Local stereology. World Scientific, Singapore.

Jensen EBV (2000). On the variance of local stereological volume estimators. Image Anal Stereol 19:15-18.

Jensen EBV, Petersen L (1999). When are local stereological volume estimators exact? Research report no. 4, Laboratory for Computational Stochastics, University of Aarhus.

Stoyan D, Kendall WS, Mecke J (1995). Stochastic geometry and its applications. 2nd ed. New York: John Wiley. 